\title{
Nonlinearity as a resource for nonclassicality in anharmonic systems
}

\author{
Francesco Albarelli, ${ }^{1}$ Alessandro Ferraro, ${ }^{2}$ Mauro Paternostro, ${ }^{2}$ and Matteo G. A. Paris ${ }^{1,3,4}$ \\ ${ }^{1}$ Dipartimento di Fisica, Università degli Studi di Milano, I-20133 Milan, Italy \\ ${ }^{2}$ School of Mathematics and Physics, Queen's University, Belfast BT7 1NN, United Kingdom \\ ${ }^{3}$ CNISM, UdR Milano Statale, I-20133 Milan, Italy \\ ${ }^{4}$ INFN, Sezione di Milano, I-20133 Milan, Italy \\ (Received 8 August 2015; published 8 March 2016)
}

\begin{abstract}
Nonclassicality is a key ingredient for quantum enhanced technologies and experiments involving macroscopic quantum coherence. Considering various exactly solvable quantum-oscillator systems, we address the role played by the anharmonicity of their potential in the establishment of nonclassical features. Specifically, we show that a monotonic relation exists between the entropic nonlinearity of the considered potentials and their ground-state nonclassicality, as quantified by the negativity of the Wigner function. In addition, in order to clarify the role of squeezing, which is not captured by the negativity of the Wigner function, we focus on the Glauber-Sudarshan $P$ function and address the nonclassicality-nonlinearity relation using the entanglement potential. Finally, we consider the case of a generic sixth-order potential confirming the idea that nonlinearity is a resource for the generation of nonclassicality and may serve as a guideline for the engineering of quantum oscillators.
\end{abstract}

DOI: 10.1103/PhysRevA.93.032112

\section{INTRODUCTION}

At the heart of quantum technologies lies the fact that quantum-mechanical systems show features, with no classical counterpart, that may be employed as resources to perform specific tasks better or faster than within the classical realm [1]. In the context of quantum optics, genuine quantum traits of optical systems have led to the emergence of the concept of nonclassicality, which characterizes states whose effects are not achievable with classical light [2]. In particular, linear models (here intended as systems that induce linear transformations of the bosonic mode operators) have attracted much attention in the past decades due to the development of experimental platforms able to implement them. In fact, the generation of nonclassical light, especially in the form of squeezed beams, has proven to be an enabling resource for a variety of quantum technological applications [3].

Recently, alternative experimental platforms have been developed that can also be coherently controlled and described as single-mode bosonic systems, including trapped ions [4], optomechanical systems [5], atoms in optical lattices [6], and hybrid systems [7]. The latter naturally embody a playground to discuss and test the generation and characterization of genuine quantum features. In particular, they offer the unique opportunity to consider nonlinear (or anharmonic) models, given that the possibility to host nonlinearities is within reach of current technologies, in particular for trapped ions [8] and optomechanical systems [9]. Interestingly, it has been shown that the inclusion of nonlinearities in the oscillator potential uncovers new possibilities to generate nonclassical states [10-18]. However, a general framework that encompasses these possibilities remains elusive, and in particular a thorough quantitative assessment of the link between nonlinearity and nonclassicality is still lacking.

The aim of this work is to investigate in detail the idea that nonlinearity is a general resource to generate nonclassicality in single-mode bosonic systems composed of anharmonic oscillators. In particular, we will focus on a quantitative assessment of the phenomenon, as we critically consider specific quantifiers of nonclassicality and nonlinearity. In fact, identifying proper measures of these quantities is crucial by itself, and in particular, different figures of merit exist that capture different features associated with nonclassicality [19-25]. The quantitative connection of the nonlinear behavior of an oscillatory system and the appearance of nonclassicality has recently been tested, in the context of nanomechanical resonators, for the Duffing oscillator model [26]. Here we extend this connection and assess its validity for more general scenarios, including three families of exactly solvable nonlinear oscillators and a generic sixth-order potential.

The remainder of this paper is structured as follows. In Sec. II we review the main conceptual tools and establish our notation and formalism. First, we introduce and discuss the two quantitative measures of nonclassicality that will be used throughout the paper, namely, entanglement potential and the volume of the negative part of the Wigner function. Then, we review a recently introduced measure to quantify the nonlinearity of a quantum oscillator [27], which in turn is based on an entropic measure of non-Gaussianity [28]. In Sec. III we analyze the quantitative connection between nonclassicality and nonlinearity for three different nonlinear potentials having an exact solution. We also highlight some differences between the two measures of nonclassicality (see also Ref. [29]). In Sec. IV we address the generic (symmetric) anharmonic potential by considering fourth- and sixth-order perturbations to the harmonic one. In Sec. V we draw our conclusions.

\section{NONCLASSICALITY OF A STATE AND NONLINEARITY OF A POTENTIAL}

\section{A. Nonclassicality of a single-mode bosonic state}

We consider a bosonic system with a single degree of freedom, such as a one-dimensional oscillator or a single mode of a bosonic field. Since we deal with single-mode systems, we will not discuss any notion related to entanglement or other nonclassical correlations. 
In the most general terms, a quantum state is said to be nonclassical if the methods of classical statistics fail to describe its properties and phenomenology. In the context of quantum optics this definition is made precise by using quasiprobability distributions in phase space. Here we are not only interested in criteria for nonclassicality, but we seek a quantitative characterization. An excellent summary on this topic can be found in the introduction of Ref. [30].

\section{P nonclassicality and entanglement potential}

According to Titulaer and Glauber [31-33], a quantum state is nonclassical when its $P$ function fails to be interpreted as a probability distribution in the phase space (see also Refs. [34-36]). It has been recently emphasized [37] that the $P$ function is the only quasiprobability distribution which can give a description that can be completely modeled using classical electrodynamics, therefore supporting the idea that to identify a classical state it is necessary to use the $P$ function. In this paper we refer to this fundamental notion as $P$-function nonclassicality, or $P$ nonclassicality for short.

The best-known way to quantify $P$ nonclassicality is the nonclassical depth [20]: It quantifies, operationally, the amount of thermal noise that is needed in order to render the $P$ function of a given state a well-behaved probability distribution and the corresponding state classical. This measure, however, is not fully suited for our purposes: in fact, although we will be interested in establishing a quantitative hierarchy of pure non-Gaussian states in terms of their nonclassicality at a set nonlinearity of a given potential, it has been proven that such states all saturate the nonclassical depth [38]; that is, they are equally and maximally nonclassical according to this measure.

This obstacle can be overcome by considering the following. It has long been known that coherent states are the only pure states that produce uncorrelated outputs when mixed by a passive linear-optics device [39]. Specifically, $P$ nonclassicality has been identified as a necessary condition for having entangled states at the output of a beam splitter [4042], and quantitative relations have been identified between nonclassicality and entanglement $[23,43-48]$ or discordlike correlations more generally [48]. The idea of quantifying the nonclassicality of a single-mode state as the two-mode entanglement at the output of a linear-optics device was introduced by Asbóth et al. [23]. In particular, it was shown that the optimal entangler is just a beam splitter with vacuum as an auxiliary state (see [49] for further discussion). By restricting ourselves to this setup, nonclassicality of the input state becomes a necessary and sufficient condition for output entanglement. As a consequence, entanglement at the output of a linear mixer may be used as a faithful quantitative measure of $P$ nonclassicality. This measure is usually referred to as the entanglement potential $\mathcal{E}(\rho)$, and it is defined as

$$
\mathcal{E}[\rho]=E\left[\hat{B}(\rho \otimes|0\rangle\langle 0|) \hat{B}^{\dagger}\right],
$$

where $\rho$ is the density matrix of the state under scrutiny, $|0\rangle$ is the vacuum state at the ancillary port of the beam splitter, $\hat{B}$ is the beam-splitter operator, and $E[\rho]$ is a suitable measure of entanglement. Our analysis will be concerned with the ground state of a given Hamiltonian model. By dealing with pure states, $E[\rho]$ can be chosen, with no ambiguity, as the entanglement entropy. This choice corresponds to the entropic entanglement potential defined in Ref. [23], which has been evaluated by expanding the wave function on the Fock space of a harmonic oscillator of unitary frequency and mass, truncating the expansion by ensuring the approximate normalization of the state before and after the beam splitter.

\section{W nonclassicality}

While the $P$ function can be a singular object, the Wigner function is always well behaved, even if it can attain negative values. Negativities of the Wigner function associated with a given state define the so-called $W$-function nonclassicality ( $W$ nonclassicalty), which is, however, only a sufficient condition for $P$ nonclassicality. It follows that there are $W$-classical states which are $P$-function nonclassical: displaced squeezed states are a remarkable example. The notion of $W$ nonclassicality has gained an operational meaning as follows: the evolution of a system which is in a $W$-nonclassical state cannot be efficiently simulated with classical resources [50,51]. In order to quantify $W$ nonclassicality we use the volume of the negative part of the Wigner function [22]

$$
\delta=\left(\int d x d p|W(x, p)|\right)-1,
$$

where $x$ and $p$ are phase-space variables and $W(x, p)$ is the Wigner function of the state under scrutiny. We will make use of the following normalized version of this measure:

$$
v=\frac{\delta}{1+\delta},
$$

which gives $v \in[0,1]$.

Let us stress that the $W$ and $P$ nonclassicalities single out different quantum features. In particular, the Hudson theorem [52] guarantees that the sole pure states with a positive Wigner function are Gaussian ones, i.e., squeezed coherent states. Hence, there exist pure states that have zero $W$ nonclassicality (e.g., squeezed states) but nonzero $P$ nonclassicality. In this sense the entanglement potential can reveal more detailed features of quantumness, as we will see below. Note that measures of $W$ nonclassicality based on the geometric distance between quantum states have also been introduced $[19,24]$.

\section{B. Quantifying the nonlinearity of a one-dimensional potential using its ground state}

The first idea to quantify the nonlinearity (intended as the anharmonicity character) of a potential would be to define a distance between potential functions and the reference harmonic potential. However, this is, in general, not feasible since potentials do not need to be integrable functions. A different approach follows from the fact that ground states and equilibrium states of anharmonic potentials are not Gaussian, as opposed to those of a quantum harmonic oscillator. We can thus choose to quantify nonlinearity by the non-Gaussianity of the ground state of a given Hamiltonian model [27]. The measure of non-Gaussianity used for this goal is the entropic measure introduced in $[28,53]$. Here we shall briefly review these measures. 


\section{Non-Gaussianity of a quantum state}

The covariance matrix of a single-mode bosonic system prepared in a state $\rho$ is defined as [54]

$$
\sigma_{j k}[\rho]=\frac{1}{2}\left\langle\left\{\hat{R}_{j}, \hat{R}_{k}\right\}\right\rangle_{\rho}-\left\langle\hat{R}_{j}\right\rangle_{\rho}\left\langle\hat{R}_{k}\right\rangle_{\rho},
$$

where $\hat{\mathbf{R}}=(\hat{x}, \hat{p})^{T}$ is the vector of single-mode quadrature operators $\hat{x}$ and $\hat{p}$ and the subscript implies that expectation values are calculated over state $\rho$. We also define the displacement vector $\overline{\mathbf{X}}[\rho]$ with components $X_{k}[\rho]=\left\langle\hat{R}_{k}\right\rangle_{\rho}$. A Gaussian state has a Gaussian Wigner function.

To quantify non-Gaussianity of a generic state $\rho$, a reference Gaussian state $\tau$ should be defined. This is identified as the Gaussian state with the same covariance matrix and displacement vector as $\rho$. That is,

$$
\overline{\mathbf{X}}[\tau]=\overline{\mathbf{X}}[\rho], \quad \sigma[\tau]=\sigma[\rho] .
$$

Non-Gaussianity can now be defined as the distance between $\rho$ and $\tau$ calculated using, for instance, the quantum relative entropy

$$
S(\rho \| \tau)=\operatorname{Tr}[\rho(\ln \rho-\ln \tau)] .
$$

We have that $S(\rho \| \tau)=0$ iff $\rho=\tau$. Although $S(\rho \| \tau)$ is not symmetric in its arguments and thus does not embody a proper metric, it has been used widely to quantify the distinguishability of two states. This leads to the definition of the entropic measure of non-Gaussianity,

$$
\delta_{\mathrm{E}}(\rho)=S(\rho \| \tau)=\operatorname{Tr}[\rho \ln \rho]-\operatorname{Tr}[\rho \ln \tau]=S(\tau)-S(\rho),
$$

where $S$ denotes the von Neumann entropy and, owing to the way $\tau$ is defined, we have that $S(\tau)=-\operatorname{Tr}[\tau \ln \tau]=$ $-\operatorname{Tr}[\rho \ln \tau]$. This measure satisfies a series of quite useful properties [28]: it is additive under the tensor product operation and invariant under symplectic transformations, which are both very useful for the sake of our analysis.

The von Neumann entropy of a single-mode Gaussian state takes the very simple form

$$
S\left(\rho_{\mathrm{G}}\right)=h(\sqrt{\operatorname{det} \sigma}),
$$

where $h(x)=\left(x+\frac{1}{2}\right) \ln \left(x+\frac{1}{2}\right)-\left(x-\frac{1}{2}\right) \ln \left(x-\frac{1}{2}\right)$. Thanks to this form, the entropic non-Gaussianity becomes

$$
\delta_{\mathrm{E}}(\rho)=h(\sqrt{\operatorname{det} \sigma})-S(\rho),
$$

which is further simplified for pure states, as $S(\rho)=0$.

\section{Nonlinearity of a potential}

We consider a generic potential $V(x)$ and denote with $|\phi\rangle$ the ground state of the corresponding Hamiltonian. The first idea to quantify nonlinearity would be again using the geometrical distance between the ground state of the potential and a reference harmonic state; in particular for this purpose the Bures metric has also been employed [27]. This way of reasoning has a downside because we have to choose a value for the frequency $\omega$ of the reference harmonic oscillator. The most natural choice is expanding the potential near its minimum and finding $\omega$ as a function of the nonlinear parameters of the potential. However, determining this frequency is not always straightforward, and for some potentials exhibiting more than one minimum it may even be misleading.

Instead of using a metric, we choose to quantify nonlinearity using the entropic non-Gaussianity $\delta_{\mathrm{E}}$, so that the measure of nonlinearity is defined as

$$
\eta_{\mathrm{NG}}[V]=\delta_{\mathrm{E}}(|\phi\rangle\langle\phi|)=h(\sqrt{\operatorname{det} \sigma})
$$

this equality holds because the ground state is pure and $\sigma$ is the covariance matrix of the ground state (we drop the dependence from the state when obvious).

This definition is more appealing than a geometric one because it does not require the determination of a reference potential for $V(x)$, just the reference Gaussian state for the ground state of $V(x)$. This makes $\eta_{\mathrm{NG}}$ independent of the specific features of the potential since we do not need to know the behavior of $V(x)$ near its minimum to compute the reference frequency.

Moreover, $\eta_{\mathrm{NG}}$ inherits the property of the non-Gaussianity measure and is invariant under symplectic transformations [54]. This means that $\delta_{\mathrm{NG}}$ assigns the same nonlinearity to oscillators which are displaced, rotated in phase space, or squeezed, which is a reasonable property for a measure of nonlinearity.

\section{EXACTLY SOLVABLE NONLINEAR OSCILLATORS}

We now analyze quantitatively the relation between the figures of merit introduced in the previous section, considering three exactly solvable anharmonic oscillators.

\section{A. Modified harmonic oscillator}

The modified harmonic oscillator (MHO) potential is defined as [55] (throughout this paper we choose units such that $\hbar=m=1$ )

$$
V_{\mathrm{MHO}}(x)=\frac{\alpha^{2} x^{2}}{2}-\alpha \beta x \tanh (\beta x) .
$$

Here $\alpha$ is a parameter corresponding to the frequency of the unmodified harmonic oscillator, while $\beta$ determines the deformation of the harmonic potential. The effects of this parameter on the shape of the potential is appreciated from Fig. 1, where $V_{\mathrm{MHO}}(x)$ is plotted at a set value of $\alpha$ for different choices of $\beta$, showing that an increasing deformation parameter transforms a harmonic potential into a double-well one whose well depth and separation both increase with $\beta$. For small $\beta$ this potential has a behavior similar to the Duffing oscillator routinely used to model nanomechanical resonators [56,57]; therefore, we think of the MHO as an extension of the Duffing model in the case of strong nonlinearity.

The normalized wave function of the ground state of this potential can be found to read [55]

$$
\phi_{\mathrm{MHO}}(x)=\frac{\sqrt{2} e^{-\frac{1}{2} \alpha x^{2}} \cosh (\beta x)}{\sqrt[4]{\frac{\pi}{\alpha}} \sqrt{1+\exp \left[\beta^{2} / \alpha\right]}} .
$$

The associated energy is $E_{0}=\left(\alpha-\beta^{2}\right) / 2$. The covariance matrix of such a least-energy state can be computed 


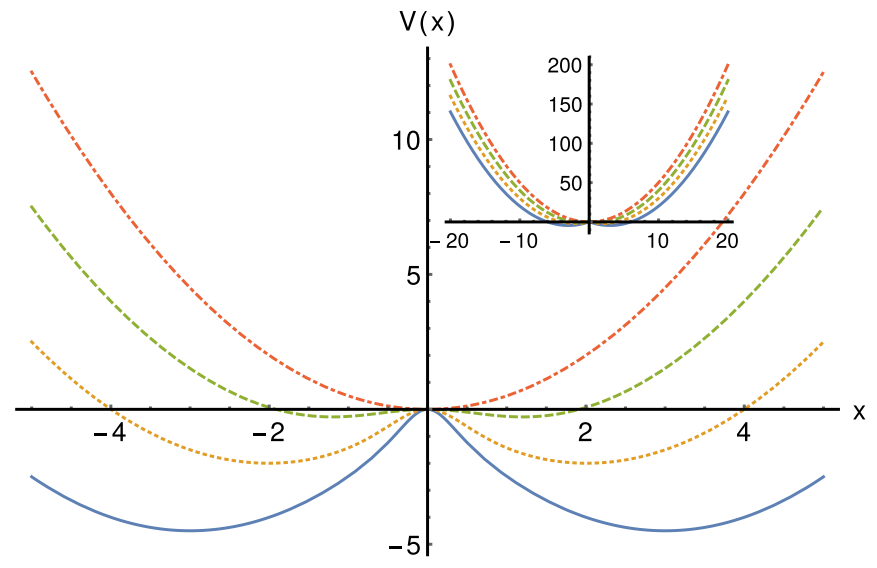

FIG. 1. The MHO potential with $\beta=3$ (solid blue line), 2 (dotted yellow line), 1 (dashed green line) and the harmonic potential with unitary frequency and mass (dot-dashed orange line); with our choice of units $x$ is measured in units of $\alpha^{-1 / 2}, V$ in units of $\alpha$, and $\beta$ in units of $\alpha^{1 / 2}$. The inset represents the same graph at a larger scale, where we see the resemblance to the harmonic potential.

straightforwardly to be

$$
\sigma^{\mathrm{MHO}}=\left(\begin{array}{cc}
\frac{1}{2 \alpha}+\frac{\beta^{2}}{\alpha^{2}} \frac{\exp \left[\beta^{2} / \alpha\right]}{1+\exp \left[\beta^{2} / \alpha\right]} & 0 \\
0 & \frac{\alpha}{2}-\frac{\beta^{2}}{1+\exp \left[\beta^{2} / \alpha\right]}
\end{array}\right) .
$$

Its determinant is

$$
\operatorname{det} \sigma^{\mathrm{MHO}}=\frac{1}{4}-\frac{\tau^{2}}{2} \frac{\left(2 \tau^{2} e^{\tau^{2}}-e^{2 \tau^{2}}+1\right)}{\left(e^{\tau^{2}}+1\right)^{2}},
$$

with the dimensionless parameter $\tau=\sqrt{\beta^{2} / \alpha}$. Such dependence on $\tau$, rather than $\alpha$ and $\beta$ independently, is common to $\eta_{\mathrm{NG}}=h(\operatorname{det} \sigma)$ and the measure of nonlinearity based on the Bures distance (for the latter, we should choose the unmodified harmonic oscillator with frequency $\alpha$ as a reference). Both measures of nonlinearity increase monotonically with $\tau$.

The Wigner function associated with $\phi_{\mathrm{MHO}}$ can be written in terms of the suitably rescaled phase-space variables $q=\beta x$ and $p=\frac{\beta}{\alpha} y$ as [55]

$$
W_{\mathrm{MHO}}(q, p)=e^{-\frac{q^{2}+p^{2}}{\tau^{2}}} \frac{\cosh (2 q)+e^{\tau^{2}} \cos (2 p)}{\pi \tau^{2}\left(1+e^{\tau^{2}}\right)},
$$

which shows again the key role played by $\tau$ and, in turn, that the nonclassicality measure based on the volume of the negative part of $W_{\mathrm{MHO}}(q, p)$ is determined by such a parameter.

In order to understand how $W$ nonclassicality and nonlinearity are related to each other, we have studied both quantities against $\tau$. In Fig. 2 we report the resulting parametric plot, showing that $v$ monotonically increases with $\eta_{\mathrm{NG}}$, thus supporting the idea that a growing degree of anharmonicity of the potential results in increased nonclassicality of the corresponding ground state.

However, the picture changes significantly as soon as we consider $P$ nonclassicality quantified by the entanglement potential, which, as said, can single out more detailed features of quantumness. Indeed, at variance with what has been found above, such a figure of merit turns out to depend on $\alpha$ and $\beta$ independently. The reason for such a difference in behavior

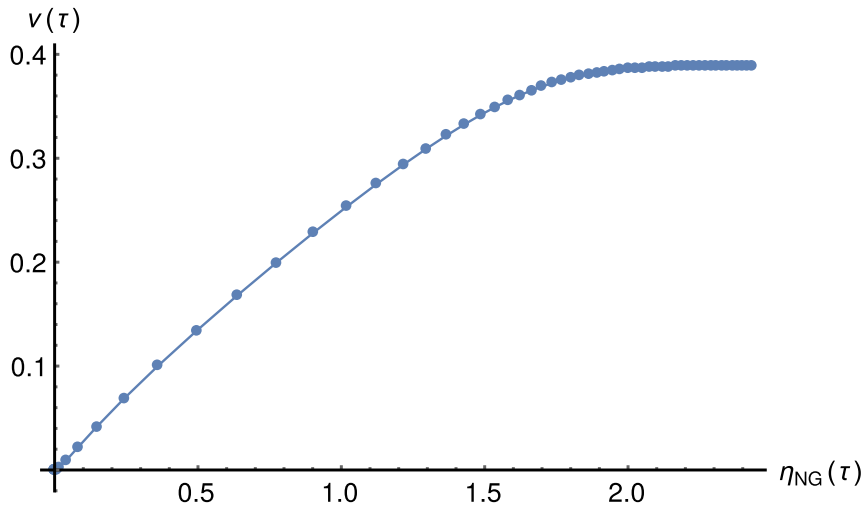

FIG. 2. Parametric plot of the $W$-nonclassicality measure $v(\tau)$ versus the degree of nonlinearity for the MHO potential and for $\tau \in[0.1,6]$.

should be ascribed to the fact that entanglement at the output of a beam splitter can be originated either by a non-Gaussian input state or by Gaussian single-mode squeezing. In other words, nonlinearity is needed to generate $W$ nonclassicality, while $P$ nonclassicality may be obtained using just squeezing.

In order to illustrate this clearly, in Fig. 3 we show the entanglement potential and squeezing for the MHO both as a function of $\beta$ for fixed values of $\tau$ and as a function of $\tau$ at set values of $\alpha$. The squeezing in Fig. 3 is shown in terms of the ratios

$$
r_{x}=\frac{\sigma_{11}^{\mathrm{MHO}}}{\sigma_{11}^{0}}=2 \sigma_{11}^{\mathrm{MHO}}, \quad r_{p}=\frac{\sigma_{22}^{\mathrm{MHO}}}{\sigma_{22}^{0}}=2 \sigma_{22}^{\mathrm{MHO}},
$$

with $\sigma_{11}^{0}=\sigma_{22}^{0}=1 / 2$ being the variances of position and momentum calculated over the vacuum state of the harmonic potential. Squeezing is found in the ground state of the MHO (a)

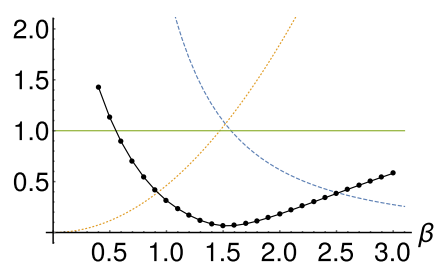

(c)

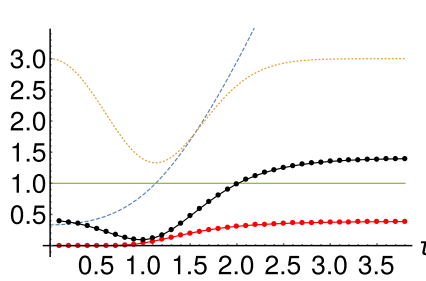

(b)

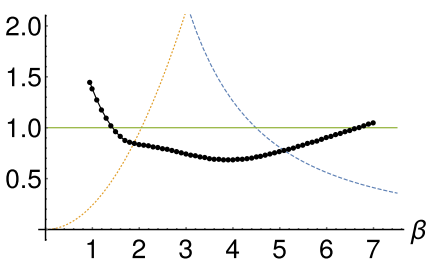

(d)

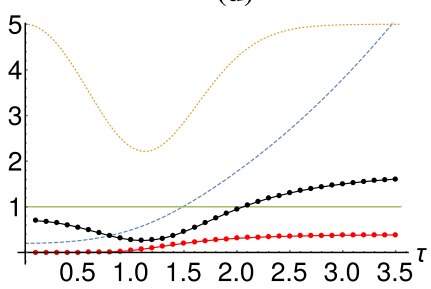

FIG. 3. Entanglement potential and squeezing for the MHO. We plot $r_{x}$ (dashed blue curve), $r_{p}$ (dotted orange curve), the entanglement potential ( $P$ nonclassicality) $\mathcal{E}$ (black dots), and $W$ nonclassicality $v$ (red dots) against (a) and (b) $\beta$ for $\tau=1$ and $\tau=3$ and (c) and (d) $\tau$ for $\alpha=3$ and $\alpha=5$. Squeezing is observed for either $r_{x}<1$ or $r_{p}<1$ (i.e., variances of the perturbed ground state below the values of the vacuum state of a harmonic oscillator). 


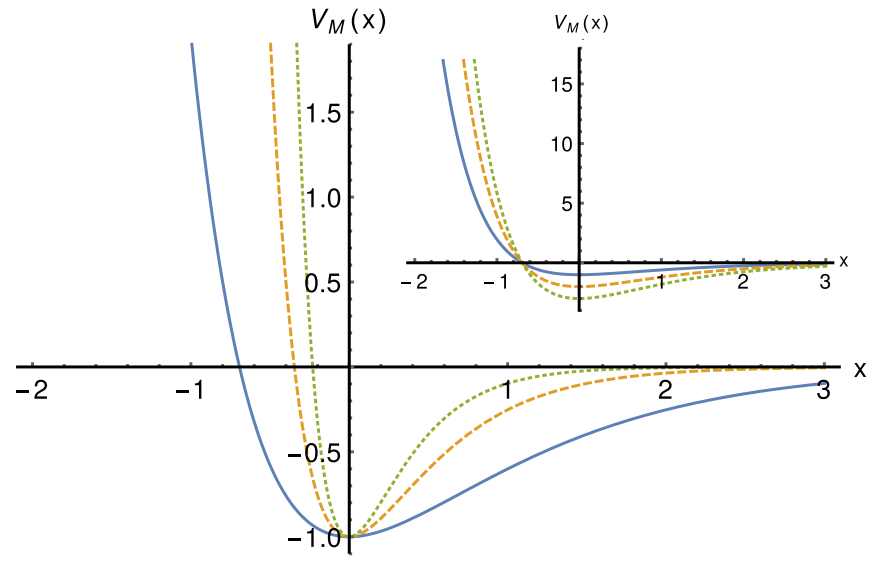

FIG. 4. The Morse potential $V_{M}(x)$ for $\alpha=1$ (solid blue line), 2 (dashed orange line), 3 (dotted green line), where $V$ is measured in units of $D, x$ in units of $D^{-1 / 2}$, and $\alpha$ in units of $D^{1 / 2}$. The inset shows the potential for $D=1$ (solid blue line), 2 (dashed orange line), 3 (dotted green line), with $x$ in units of $\alpha^{-1}$ and $V$ and $D$ in units of $\alpha^{2}$

for either $r_{x}<1$ or $r_{p}<1$. As is apparent from Fig. 3, the behavior of $\mathcal{E}$ is rather different from that of $v$, and its features may be understood by looking at squeezing. In particular, we see that $\mathcal{E}$ grows when the ground state exhibits squeezing.

\section{B. Morse potential}

The Morse potential has been introduced as an approximation to the potential energy of diatomic molecules as it provides a better description of the vibrational structure than the (quantum) harmonic oscillator [58]. The form of the potential is

$$
V_{\mathrm{M}}=D\left(e^{-2 \alpha x}-2 e^{-\alpha x}\right),
$$

where $x$ is the distance from the minimum of the potential, the parameter $D>0$ determines the depth of the well, and $\alpha$ controls its width. Expanding the two exponentials for $\alpha \rightarrow 0$ at fixed $D$, we get the harmonic limit, which is an oscillator with frequency $\omega_{\mathrm{M}}=\sqrt{2 D} \alpha$. The potential is plotted in Fig. 4 for different values of the parameters.

The Schrödinger equation associated with this potential can be solved analytically, with the energy eigenvectors being labeled by two quantum numbers, which we label here $N$ and $v$. The first is related to the parameters of the potential as $N=-1 / 2+\sqrt{2 D} / \alpha$. The second, which can take values $v=$ $0,1,2, \ldots,\lfloor N\rfloor$, counts the number of anharmonic excitations of the system. As we want at least one bound state, we require $N>0$. We thus have the constraint $\alpha<2 \sqrt{2 D}$. The limiting case where we have just one bound state (the ground state) is achieved for $\alpha \rightarrow 2 \sqrt{2 D}$. The wave function of the ground state is

$$
\phi_{\mathrm{M}}(x)=(2 N+1)^{N} \sqrt{\frac{\alpha}{(N-1) !}} e^{-\alpha x N-\left(N+\frac{1}{2}\right) e^{-\alpha x}},
$$

with associated energy $E=-\alpha N^{2} / 2$. The behavior of the nonlinearity of the Morse potential can be understood by looking at the form of the potential in Fig. 4, as opposed to the harmonic one [27]: For any fixed value of $D(\alpha)$ we expect an increase (decrease) of nonlinearity for increasing $\alpha(D)$.

The covariance matrix associated with the ground state in Eq. (18) is

$$
\sigma^{\mathrm{M}}=\left(\begin{array}{cc}
\frac{\psi^{(1)}(2 N)}{\alpha^{2}} & 0 \\
0 & \frac{\alpha^{2} N}{2}
\end{array}\right)
$$

where $\psi^{(n)}(z)$ is the polygamma function $\psi^{(n)}(z)=$ $\frac{\mathrm{d}^{n+1}}{\mathrm{~d} z^{n+1}} \log \Gamma(z)$, with $\Gamma(Z)$ being the Euler gamma function. The determinant of this correlation matrix, as well as the Bures distance from the reference harmonic oscillator, depends only on $N$, or, equivalently, on the combination $\sqrt{2 D} / \alpha$. In this case both measures of nonlinearity are monotonically decreasing functions of $N$.

The Wigner function for the ground state of the Morse potential reads [59]

$$
W_{\mathrm{M}}(x, p)=\frac{2(2 N+1)^{2 N}}{\pi \Gamma(2 N)} e^{-2 N \alpha x} K_{-2 I p / \alpha}\left[(2 N+1) e^{-\alpha x}\right],
$$

where $K_{\gamma}(z)$ is the Macdonald function of (noninteger) order $\gamma$. In order to calculate the measure of nonclassicality $v$, we rescale the phase-space variables to $q=\alpha x$ and $p=\frac{y}{\alpha}$ and evaluate

$$
\begin{aligned}
\iint d x d y\left|W_{\mathrm{M}}(x, y)\right|= & \iint d q d p \mid \frac{2 e^{-2 N q}}{\pi \Gamma(2 N)}(2 N+1)^{2 N} \\
& \times K_{-2 I p}\left[(2 N+1) e^{-q}\right] \mid
\end{aligned}
$$

which shows that the only relevant parameter is $N$. The numerical integration of Eq. (21) is challenging and was carried out with the aid of the CUBA libraries [60]. The degree of $W$ nonclassicality $v$ is found to monotonically decreases with $N$, and the parametric plot of nonclassicality versus nonlinearity in Fig. 5 reveals a monotonic behavior, strengthening the link between such features and reinforcing the idea that nonlinearity might play the role of a catalyst for nonclassicality.

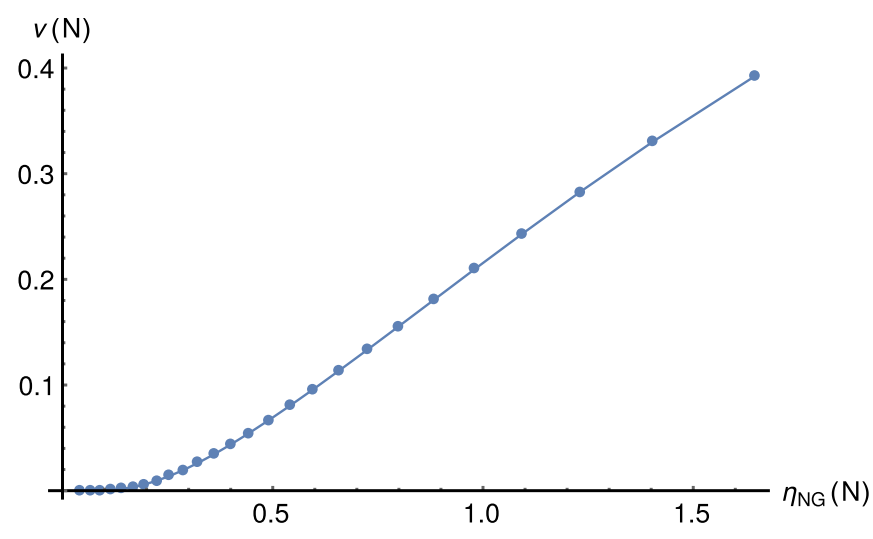

FIG. 5. Parametric plot of the $W$ nonclassicality $v$ versus the degree of nonlinearity $\eta$ for a Morse potential with $D=1$ and $\alpha \in$ $[0.15,2.7]$, i.e., $N \in[0.0238,8.928]$. 
(a)

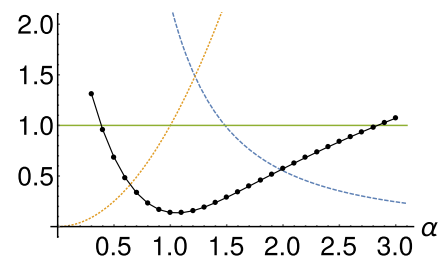

(c)
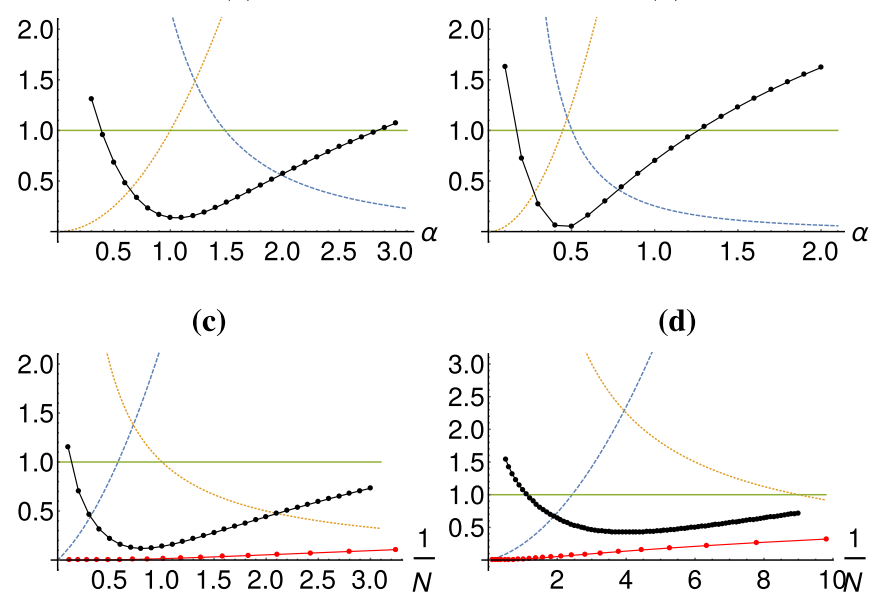

(d)

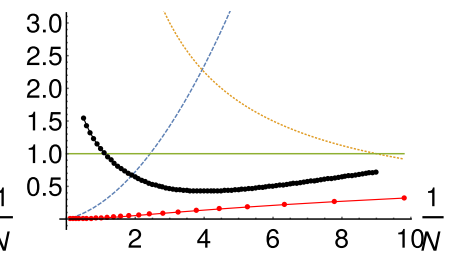

FIG. 6. Entanglement potential and squeezing for the Morse oscillator. We plot $r_{x}$ (dashed blue curve), $r_{p}$ (dotted orange curve), the entanglement potential ( $P$ nonclassicality) $\mathcal{E}$ (black dots), and $W$ nonclassicality $v$ (red dots) against (a) and (b) $\alpha$ for $N=1$ and $N=5$ and (c) and (d) $1 / N$ for $\alpha=1$ and $\alpha=3$. Squeezing is observed for either $r_{x}<1$ or $r_{p}<1$ (i.e., variances of the perturbed ground state below the values of the vacuum state of a harmonic oscillator).

The situation regarding the entanglement potential is completely analogous to what we found for the MHO, as it depends on both parameters. In Fig. 6 we report the same kind of graphs, with both $N$ and $\alpha$ fixed, which show that the behavior of $\mathcal{E}$ is explained by the squeezing of the state.

\section{Pöschl-Teller potential}

The Pöschl-Teller (PT) potential has been studied since the early days of quantum mechanics (see, e.g., [61]); it has been applied in the context of semiconductor quantum wells [62$64]$, and it can be used to model nonlinear optical properties $[65,66]$.

In particular we will use the modified PT potential, defined as

$$
V_{\mathrm{PT}}(x)=-A_{\mathrm{PT}} \cosh ^{-2}(\alpha x),
$$

where $A_{\mathrm{PT}}>0$ is the depth of the potential and $\alpha$ is connected to its range. The harmonic limit is obtained at fixed $A_{\mathrm{PT}}$ for $\alpha \rightarrow 0$, and the frequency of the reference harmonic oscillator is $\omega_{\mathrm{PT}}=\sqrt{2 A_{\mathrm{PT}}} \alpha$. As for the Morse potential, we have a quantum number $s$ that labels the energy eigenstates and counts the anharmonic excitations. It is related to the parameters of the potential through the relation $A_{\mathrm{PT}}=\frac{1}{2} \alpha^{2} s(s+1)$. Therefore, the request for the existence of at least one bound state translates into $s=\frac{1}{2}\left(-1+\sqrt{1+8 A_{\mathrm{PT}} / \alpha^{2}}\right)>0$. Figure 7 shows the dependence of the PT potential on the position coordinate.

The ground state of the system reads

$$
\phi_{\mathrm{PT}}(x)=\frac{1}{\pi^{\frac{1}{4}}} \sqrt{\frac{\alpha \Gamma\left(s+\frac{1}{2}\right)}{\Gamma(s)}} \cosh ^{-s}(\alpha x),
$$

with associated energy $E=-\alpha^{2} s^{2} / 2$.

Different from the previous cases, the covariance matrix of the ground state is rather involved and will not be reported

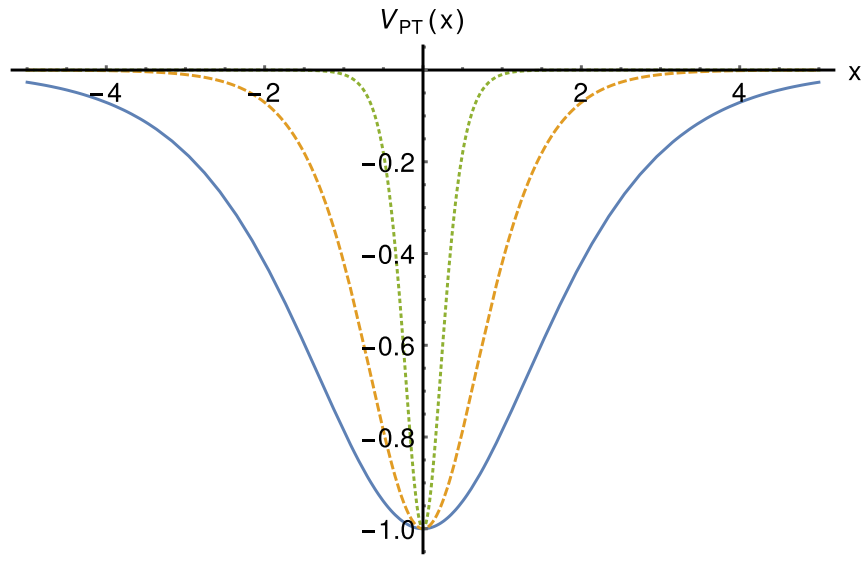

FIG. 7. The Posh-Teller potential with $\alpha=1 / 2$ (solid blue line), 1 (dashed orange line), and 3 (dotted green line), with $x$ in units of $A_{\mathrm{PT}}^{-1 / 2}, \alpha$ in units of $A_{\mathrm{PT}}^{1 / 2}$, and $V_{\mathrm{PT}}$ in units of $A_{\mathrm{PT}}$.

here; it can, however, be easily obtained by directly applying $x$ and $p$ operators in the position representation on the wave function (23).

In line with the case of the previous two anharmonic potentials studied here, its determinant depends only on $s$ (or, equivalently, on $A_{\mathrm{PT}} / \alpha^{2}$ ). Again, both $\eta_{\mathrm{NG}}$ and the Bures nonlinearity are monotonically decreasing functions of only $s$.

The Wigner function of state $\phi_{\mathrm{PT}}(x)$ in Eq. (23) is known analytically for the case of $A_{\mathrm{PT}}=\alpha^{2}$ [55]. In this case, the measure $v$ is an $s$-dependent constant, which can be seen by rescaling the relevant variables as $p^{\prime}=\frac{p}{\alpha}, x^{\prime}=\alpha x, y^{\prime}=\alpha y$ and evaluating the integral

$$
W_{\mathrm{PT}}(x, p)=\int d y \phi_{\mathrm{PT}}^{*}\left(x-\frac{y}{2}\right) \phi_{\mathrm{PT}}\left(x+\frac{y}{2}\right) e^{-I y p},
$$

which embodies the definition of the Wigner function.

As for the entanglement potential, this turns out to depend on both $\alpha$ and $s$. Plots similar to those valid for the MHO and Morse potential are presented in Fig. 8 (without the $W$ nonclassicality $v)$. (a)

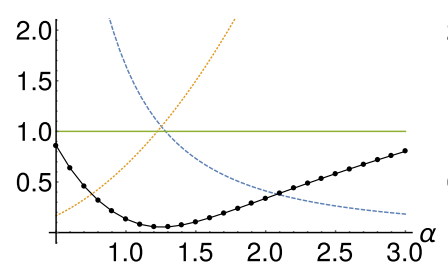

(c)

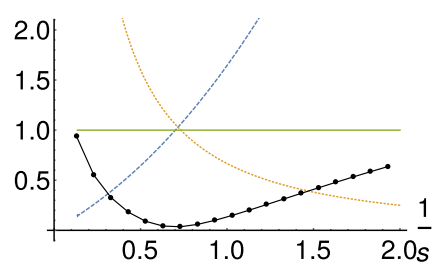

(b)

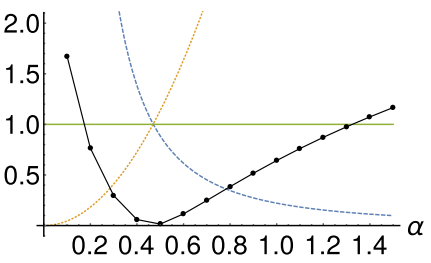

(d)

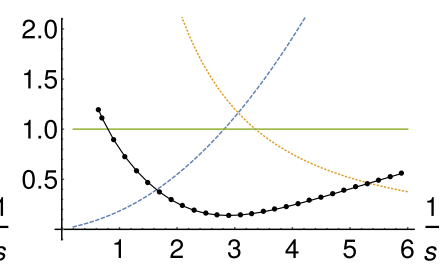

FIG. 8. Entanglement potential and squeezing for the PT oscillator. We show $r_{x}$ (dashed blue curve), $r_{p}$ (dotted orange curves), and the entanglement potential $\mathcal{E}$ (black dots) against (a) and (b) $\alpha$ for $s=1$ and $s=5$ and (c) and (d) $1 / s$ for $\alpha=1$ and $\alpha=3$. 


\section{OSCILLATORS WITH POLYNOMIAL PERTURBATIONS}

So far we have studied exactly solvable potentials with two parameters and revealed a common behavior: the nonlinearity and the $W$ nonclassicality $v$ have the same behavior and depend just on a single effective parameter. On the other hand, the entanglement potential carries a dependence on both the parameters, and its different behavior may be understood in terms of the squeezing of the state.

Now we want to address the case of a generic two-parameter perturbation, so we study a physical system composed of a one-dimensional harmonic oscillator with perturbations proportional to $x^{4}$ and $x^{6}$, respectively. The Hamiltonian of this system thus reads

$$
H=\frac{1}{2}\left(p^{2}+\omega^{2} x^{2}\right)+\epsilon_{4} x^{4}+\epsilon_{6} x^{6} .
$$

As the model is not exactly solvable, the properties of the system will be studied using perturbation theory. We notice that Eq. (25) may also serve as an approximation for any symmetric (even) potential. In particular this Hamiltonian can also be intuitively considered as a generalization of the static Duffing oscillator.

We do not consider odd powers of $x$ or negative coefficients for the even powers of $x$, even when the potential is still bounded from below. We make this choice in order to avoid any ambiguity, which could arise when the potential has more than one minimum. In fact, for such a case, the state obtained with the perturbative expansion is not necessarily an approximation to the true ground state but could be a state associated with a local minimum of energy.

A remark is in order: terms proportional to $x$ and to $x^{2}$ could, in principle, be treated in a perturbative way as well. However, they do not give rise to truly anharmonic behavior and will not be considered in this context.

In order to get insight into the ground states for these Hamiltonians we use first-order time-independent perturbation theory [67]. The state takes the form

$$
|\psi\rangle=\sum_{n=0}^{3} \gamma_{2 n}|2 n\rangle
$$

where $|k\rangle$ denotes a Fock number state of the harmonic oscillator and the coefficients $\gamma_{k}$ are in given Sec. A 1.

Applying first-order perturbation theory, the ground state can be approximated with a finite superposition of Fock states, which makes the Wigner function and the nonlinearity easy to compute. In order to assess the validity of the first-order approximation, we compare such a ground state to the state obtained by numerically diagonalizing the Hamiltonian of the system within a truncated Fock space of suitable size. Convergence of the results of such numerical calculations appears to be ensured by using 61 harmonic levels. The corresponding ground state $|\phi\rangle$ is then compared to $|\psi\rangle$ using the state fidelity $|\langle\phi \mid \psi\rangle|^{2}$. In Fig. 9 we present a contour plot of the overlap as a function of both $\epsilon_{4}$ and $\epsilon_{6}$. For values of $\epsilon_{4}$ up to 0.1 and $\epsilon_{6}$ up to 0.03 the fidelity is at least $\approx 0.976$.

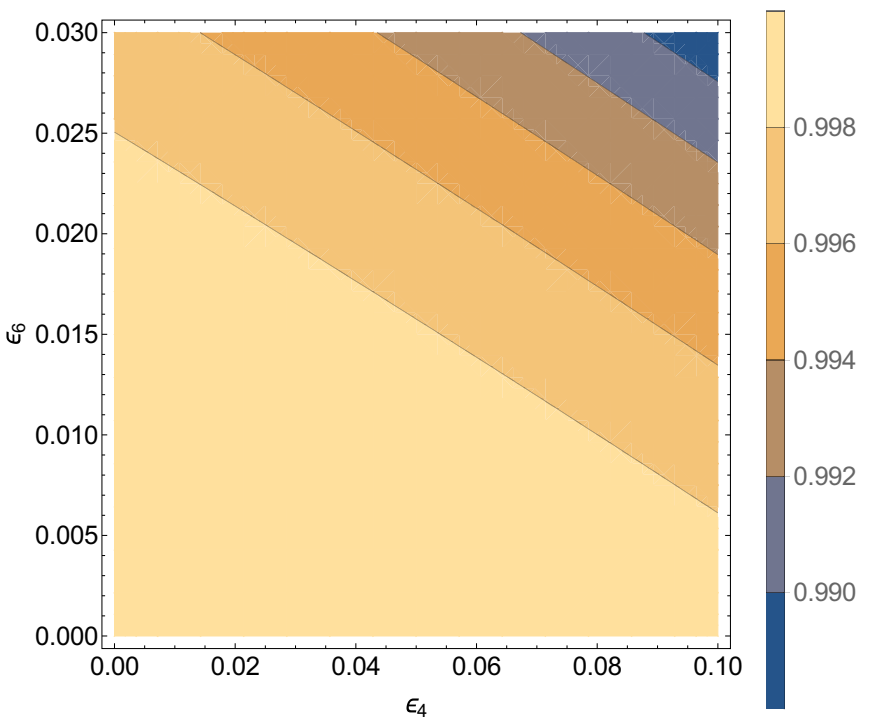

FIG. 9. Contour plot of the overlap between the perturbative ground states of Eq. (26) and the numerically calculated one (for $\omega=1)$.

\section{Nonclassicality and nonlinearity}

From the perturbed ground state in Eq. (26) we compute the nonlinearity of the perturbing potential. The covariance matrix associated with $|\psi\rangle$ can be thus written as

$$
\sigma^{\mathrm{pol}}=\left(\begin{array}{cc}
\frac{\left(1+2\left\langle\hat{a}^{2}\right\rangle+2\left\langle\hat{a}^{\dagger} a\right\rangle-4\langle\hat{a}\rangle^{2}\right)}{2 \omega} & 0 \\
0 & \frac{\omega}{2}\left(1+2\left\langle\hat{a}^{\dagger} \hat{a}\right\rangle-2\left\langle\hat{a}^{2}\right\rangle\right)
\end{array}\right),
$$

with $\hat{a}$ and $\hat{a}^{\dagger}$ being the annihilation and creation operators of the oscillator and

$$
\begin{aligned}
\left\langle\hat{a}^{\dagger}\right\rangle & =\langle\hat{a}\rangle=0, \\
\left\langle\hat{a}^{\dagger} a\right\rangle & =2\left|\gamma_{2}\right|^{2}+4\left|\gamma_{4}\right|^{2}+6\left|\gamma_{4}\right|^{2}, \\
\left\langle\hat{a}^{2}\right\rangle & =\sqrt{2} \gamma_{2} \gamma_{0}{ }^{*}+2 \sqrt{3} \gamma_{4} \gamma_{2}{ }^{*}+\sqrt{30} \gamma_{6} \gamma_{4}{ }^{*}, \\
\left\langle\hat{a}^{\dagger 2}\right\rangle & =\left\langle\hat{a}^{2}\right\rangle^{*}=\sqrt{2} \gamma_{0} \gamma_{2}{ }^{*}+2 \sqrt{3} \gamma_{2} \gamma_{4}{ }^{*}+\sqrt{30} \gamma_{4} \gamma_{6}{ }^{*} .
\end{aligned}
$$

An explicit calculation shows that the determination of $\sigma^{\mathrm{pol}}$, and in turn the nonlinearity $h(\sqrt{\operatorname{det} \sigma})$, depends on both the perturbative parameters and the frequency $\omega$. No singleparameter rescaling can be identified in this case, thus entailing the double dependence highlighted above, which is passed to the $W$ nonclassicality $v$ (see Sec. A 1 ).

As our aim is to highlight the role played by the perturbative parameters; in the remainder of our analysis we set $\omega=1$ and generate random pairs of values $\left(\epsilon_{4}, \epsilon_{6}\right)$ (within the appropriate range of validity of the first-order perturbative approach discussed above) that are then used to compute both the nonclassicality and nonlinearity indicators.

The results shown in Figs. 10 and 11 showcase a nonmonotonic relation between nonlinearity and nonclassicality: the points corresponding to the randomly taken pairs of values for the parameters are distributed within a (narrow) region comprised within four curves, each associated with an extremal value of $\epsilon_{4,6}$.

Nonclassicality and nonlinearity are thus strongly dependent on the details of the system under consideration and are, 


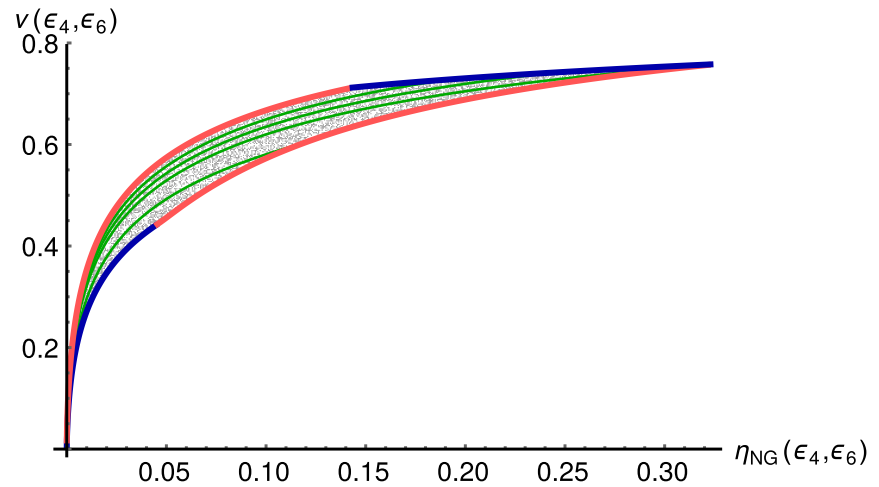

FIG. 10. Random scatter plot of the $W$ nonclassicality $v$ versus the nonlinearity $\eta_{\mathrm{NG}}$ for the perturbed harmonic oscillator when both parameters $\epsilon_{4}$ and $\epsilon_{6}$ are varied in the range given in Fig. 9; $10^{4}$ random points were generated. The dark blue curve below the points represents $\epsilon_{6}=0$, while the one above the points is the curve for $\epsilon_{6}=0.03$. The light red curve below the points is the one for $\epsilon_{4}=0.1$, while the one above the points is for $\epsilon_{4}=0$. The green curves in the middle are obtained by choosing $\epsilon_{6}=k \epsilon_{4}$; from top to bottom they correspond to the values $k=2,1,0.5,0.3,0.1$.

strictly speaking, nonequivalent notions. On the other hand, the regions in Figs. 10 and 11 are concentrated enough to suggest that the intuitive link between such two features is, in fact, correct. Moreover, if there is only one effective parameter, by fixing the value of either $\epsilon_{4}$ or $\epsilon_{6}$ or by keeping their ratio fixed (i.e., $\epsilon_{6}=k \epsilon_{4}$ ), the behavior of both nonclassicality measures becomes monotonic with nonlinearity.

Digging into the details of the phenomenological behavior identified by our analysis, it appears that $W$ nonclassicality (Fig. 10) is favored by the $x^{6}$-like nonlinearity. On the other hand, $P$ nonclassicality appears to benefit from an $\hat{x}^{4}$-type of nonlinear effects: in Fig. 11 the roles of the dark blue and light red curves are inverted with respect to Fig. 10, showing that, after choosing the parameters $\epsilon_{4}$ and $\epsilon_{6}$ in such a way that the

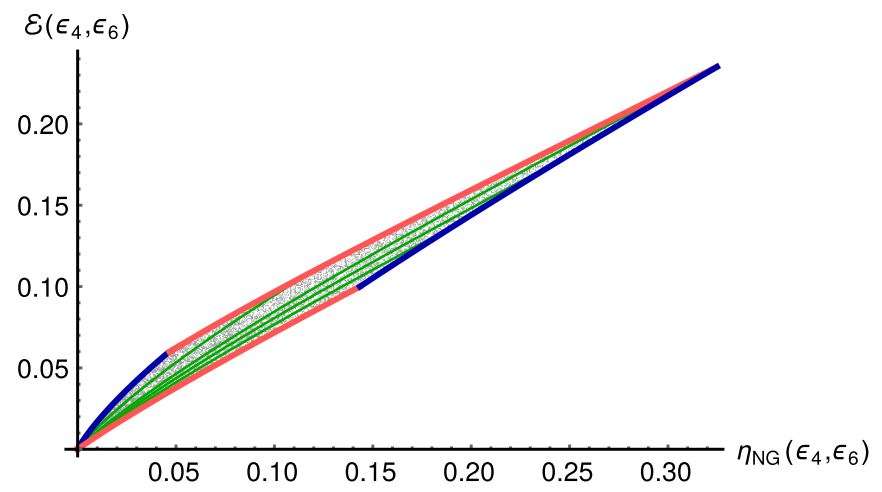

FIG. 11. Random scatter plot of the entanglement potential ( $P$ nonclassicality) $\mathcal{E}$ versus the nonlinearity $\eta_{\mathrm{NG}}$ for the perturbed harmonic oscillator when both parameters $\epsilon_{4}$ and $\epsilon_{6}$ are varied in the range given in Fig. 9; $10^{4}$ random points were generated. The dark blue curve above the points represents $\epsilon_{6}=0$, while the one below the points is the curve for $\epsilon_{6}=0.03$. The light red curve above the points is the one for $\epsilon_{4}=0.1$, while the one below the points is for $\epsilon_{4}=0$. The green curves in the middle are obtained by choosing $\epsilon_{6}=k \epsilon_{4}$; from top to bottom they correspond to the values $k=2,1,0.5,0.3,0.1$. entropic nonlinearity is fixed, the ground state obtained with the maximum value of $\epsilon_{4}$ generates more entanglement than any other one.

\section{CONCLUSIONS}

We have addressed in detail the role played by the nonlinearity of anharmonic potentials in the generation of nonclassicality in their ground states. In particular, we have shown that nonlinearity plays a crucial role in the generation of $W$ nonclassicality, while $P$ nonclassicality may also be obtained by potential inducing just squeezing.

Our results support the expectation, put forward in Ref. [26], that the nonlinearity of a potential is quantitatively related to the nonclassicality of its ground state, and thus, the former feature may be regarded as a resource to generate the latter one. The strict validity of such an expectation, which appears to be conceptually quite intuitive, is, however, strongly linked to the specific details of the Hamiltonian model being addressed. The solvable anharmonic potentials we considered can be reduced to a single-parameter dependence and give rise, in fact, to a monotonic relation between nonlinearity and $W$ nonclassicality. Such a correspondence breaks down for effectively multiparameter potentials: set values of nonlinearity bound the possible degrees of nonclassicality of the ground state of a given anharmonic potential, albeit without determining it unambiguously.

Our investigation opens up a series of questions, all linked to the effective role that nonharmonic oscillators might have in the quantum technology arena: it would be interesting, for instance, to investigate whether the enhanced nonclassicality achieved, in general, for a non-null degree of nonlinearity is accompanied by an equally enhanced degree of coherence in the ground state of the oscillator. Equally interesting is the question on the actual use that can be made of the sought nonlinearity in protocols of practical quantum estimation.

\section{ACKNOWLEDGMENTS}

This work was supported by the UK EPSRC (EP/M003019/1), the John Templeton Foundation (Grant No. 43467), the EU through the Collaborative Projects QuProCS (Grant Agreement No. 641277) and TherMiQ (Grant Agreement No. 618074), and UniMI through the H2020 Transition Grant No. 15-6-3008000-625.

\section{APPENDIX: CALCULATIONS FOR THE HARMONIC OSCILLATOR WITH PERTURBATIONS}

\section{Perturbative states}

The matrix elements of the two perturbations on the basis of the energy eigenstates of the unperturbed system, which in this case are the Fock states $|n\rangle$, are

$$
\begin{gathered}
\left\langle n\left|\hat{x}^{4}\right| n\right\rangle=\frac{6 n^{2}+6 n+3}{4 \omega^{2}}, \\
\left\langle n\left|\hat{x}^{4}\right| n+4\right\rangle=\frac{\sqrt{(n+1)(n+2)(n+3)(n+4)}}{4 \omega^{2}}, \\
\left\langle n\left|\hat{x}^{4}\right| n+2\right\rangle=\frac{(4 n+6) \sqrt{n(n-1)}}{4 \omega^{2}}
\end{gathered}
$$


for $x^{4}$ and

$$
\begin{gathered}
\left\langle n\left|\hat{x}^{6}\right| n\right\rangle=\frac{5\left(4 n^{3}+6 n^{2}+8 n+3\right)}{8 \omega^{3}}, \\
\left\langle n\left|\hat{x}^{6}\right| n+6\right\rangle \\
=\frac{\sqrt{(n+1)(n+2)(n+3)(n+4)(n+5)(n+6)}}{8 \omega^{3}},
\end{gathered}
$$

$$
\left\langle n\left|\hat{x}^{6}\right| n+4\right\rangle=\frac{3(2 n+5) \sqrt{(n+1)(n+2)(n+3)(n+4)}}{8 \omega^{3}},
$$

$$
\left\langle n\left|\hat{x}^{6}\right| n+2\right\rangle=\frac{15\left(n^{2}+3 n+3\right) \sqrt{(n+1)(n+2)}}{8 \omega^{3}}
$$

for $x^{6}$, and all the other elements are zero apart from the symmetrical ones (i.e., $\left\langle n\left|\hat{x}^{4}\right| n+k\right\rangle=\left\langle n+k\left|\hat{x}^{4}\right| n\right\rangle$ and $\left.\left\langle n\left|\hat{x}^{6}\right| n+k\right\rangle=\left\langle n+k\left|\hat{x}^{6}\right| n\right\rangle\right)$.

The formula for the perturbed ground state is

$$
|\psi\rangle=|0\rangle+\epsilon \sum_{k \neq 0}|k\rangle \frac{V_{k 0}}{-\omega k}
$$

where $V_{n k}=\left\langle n^{(0)}|V| k^{(0)}\right\rangle$ and $V$ stands for the perturbation $\epsilon_{4} x^{4}+\epsilon_{6} x^{6}$. Using this formula and the matrix elements (A1)(A7), we readily find the normalized ground state (26); its coefficients are

$$
\begin{aligned}
& \gamma_{0}=\frac{1}{C}, \quad \gamma_{2}=-\frac{\gamma_{0}}{\sqrt{2}}\left(\frac{45 \epsilon_{6}}{4 \omega^{3}}+\frac{3 \epsilon_{4}}{\omega^{2}}\right), \\
& \gamma_{4}=-\gamma_{0} \sqrt{\frac{3}{2}}\left(\frac{15 \epsilon_{6}}{2 \omega^{3}}+\frac{\epsilon_{4}}{\omega^{2}}\right), \quad \gamma_{6}=-\sqrt{5} \gamma_{0} \epsilon_{6},
\end{aligned}
$$

where the normalization constant $C$ is

$$
C=\frac{\sqrt{\omega^{2}\left(96 \omega^{6}+117 \epsilon_{4}^{2}\right)+945 \omega \epsilon_{4} \epsilon_{6}+2055 \epsilon_{6}^{2}}}{4 \sqrt{6} \omega^{4}} .
$$

\section{Wigner function}

It is convenient to express the Wigner function as $W(\alpha)=$ $\frac{2}{\pi} \operatorname{Tr}\left[\rho \hat{D}(2 \alpha)(-1)^{\hat{a}^{\dagger} \hat{a}}\right][68,69]$, where $\hat{D}$ is the displacement operator $\hat{D}(\xi)=\exp \left(\xi \hat{a}^{\dagger}-\xi^{*} \hat{a}\right)$. The expectation values of $\hat{D}$ on Fock states are given by

$\left\langle n^{\prime}|D(z)| n\right\rangle=\left\{\begin{array}{lll}\sqrt{\frac{n !}{n^{\prime} !}} e^{-\frac{|z|^{2}}{2}}(-z)^{n^{\prime}-n} L_{n^{\prime}}^{\left(n^{\prime}-n\right)}\left(|z|^{2}\right) & \text { if } & n^{\prime}>n, \\ \sqrt{\frac{n^{\prime} !}{n !}} e^{-\frac{|z|^{2}}{2}}\left(z^{*}\right)^{n-n^{\prime}} L_{n}^{\left(n-n^{\prime}\right)}\left(|z|^{2}\right) & \text { if } & n>n^{\prime},\end{array}\right.$

where $L_{n}^{(\alpha)}(x)$ are the associated Laguerre polynomials. The Wigner function then becomes

$$
\begin{aligned}
W(z)= & \frac{2}{\pi} e^{-2|z|^{2}}\left[\gamma_{0}^{2} L_{0}\left(4|z|^{2}\right)+\gamma_{2}^{2} L_{2}\left(4|z|^{2}\right)+\gamma_{4}^{2} L_{4}\left(4|z|^{2}\right)\right. \\
& +\gamma_{6}^{2} L_{6}\left(4|z|^{2}\right)+4 \sqrt{2} \gamma_{0} \gamma_{2} \operatorname{Re}\left(z^{2}\right) L_{2}^{2}\left(4|z|^{2}\right) \\
& +\frac{16}{\sqrt{3}} \gamma_{0} \gamma_{4} \operatorname{Re}\left(z^{4}\right) L_{4}^{4}\left(4|z|^{2}\right)+\frac{32}{3 \sqrt{5}} \gamma_{0} \gamma_{6} \operatorname{Re}\left(z^{6}\right) \\
& \times L_{6}^{6}\left(4|z|^{2}\right)+\frac{4}{\sqrt{3}} \gamma_{2} \gamma_{4} \operatorname{Re}\left(z^{2}\right) L_{4}^{2}\left(4|z|^{2}\right) \\
& +\frac{16}{3 \sqrt{10}} \gamma_{2} \gamma_{6} \operatorname{Re}\left(z^{4}\right) L_{6}^{4}\left(4|z|^{2}\right) \\
& \left.+\frac{8}{\sqrt{30}} \gamma_{4} \gamma_{6} \operatorname{Re}\left(z^{2}\right) L_{6}^{2}\left(4|z|^{2}\right)\right],
\end{aligned}
$$

where the coefficients are given by (A9).
[1] A. Nielsen and I. L. Chuang, Quantum Computation and Quantum Information (Cambridge University Press, Cambridge, 2010).

[2] L. Mandel and E. Wolf, Optical Coherence and Quantum Optics (Cambridge University Press, Cambridge, 1995).

[3] For a recent review, see A. I. Lvovsky, in Fundamentals of Photonics and Physics, Photonics Vol. 1 (Wiley, West Sussex, UK, 2015), pp. 121-164.

[4] D. Leibfried, R. Blatt, C. Monroe, and D. Wineland, Rev. Mod. Phys. 75, 281 (2003).

[5] M. Aspelmeyer, T. J. Kippenberg, and F. Marquardt, Rev. Mod. Phys. 86, 1391 (2014).

[6] M. Lewenstein, A. Sanpera, and V. Ahufinger, Ultracold Atoms in Optical Lattices: Simulating Quantum Many-Body Systems (Oxford University Press, Oxford, 2013).

[7] B. Rogers, N. Lo Gullo, G. De Chiara, G. M. Palma, and M. Paternostro, Quantum Meas. Quantum Metrol. 2, 11 (2014).

[8] J. P. Home, D. Hanneke, J. D. Jost, D. Leibfried, and D. J. Wineland, New J. Phys. 13, 073026 (2011).

[9] J. C. Sankey, C. Yang, B. M. Zwickl, A. M. Jayich, J. G. E. Harris, Nat. Phys. 6, 707 (2010).

[10] V. Peano and M. Thorwart, New J. Phys. 8, 21 (2006).
[11] A. Kolkiran and G. S. Agarwal, arXiv:cond-mat/0608621.

[12] C. Joshi, M. Jonson, E. Andersson, and P. Öhberg, J. Phys. B 44, 245503 (2011).

[13] F. R. Ong, M. Boissonneault, F. Mallet, A. Palacios-Laloy, A. Dewes, A. C. Doherty, A. Blais, P. Bertet, D. Vion, and D. Esteve, Phys. Rev. Lett. 106, 167002 (2011).

[14] D. P. DiVincenzo and J. A. Smolin, New J. Phys. 14, 013051 (2012).

[15] S. Rips and M. J. Hartmann, Phys. Rev. Lett. 110, 120503 (2013).

[16] S. Rips, M. Kiffner, I. Wilson-Rae, and M. J. Hartmann, New J. Phys. 14, 023042 (2012).

[17] G. Vacanti, M. Paternostro, G. M. Palma, M. S. Kim, and V. Vedral, Phys. Rev. A 88, 013851 (2013).

[18] V. Montenegro, A. Ferraro, and S. Bose, Phys. Rev. A 90, 013829 (2014).

[19] M. Hillery, Phys. Rev. A 35, 725 (1987).

[20] C. T. Lee, Phys. Rev. A 44, R2775(R) (1991).

[21] T. Richter and W. Vogel, Phys. Rev. Lett. 89, 283601 (2002).

[22] A. Kenfack and K. Życzkowski, J. Opt. B 6, 396 (2004).

[23] J. K. Asbóth, J. Calsamiglia, and H. Ritsch, Phys. Rev. Lett. 94, $173602(2005)$. 
[24] A. Mari, K. Kieling, B. M. Nielsen, E. S. Polzik, and J. Eisert, Phys. Rev. Lett. 106, 010403 (2011).

[25] A. Ferraro and M. G. A. Paris, Phys. Rev. Lett. 108, 260403 (2012).

[26] B. Teklu, A. Ferraro, M. Paternostro, and M. G. A. Paris, EPJ Quantum Technol. 2, 16 (2015).

[27] M. G. A. Paris, M. G. Genoni, N. Shammah, and B. Teklu, Phys. Rev. A 90, 012104 (2014).

[28] M. G. Genoni and M. G. A. Paris, Phys. Rev. A 82, 052341 (2010).

[29] J. Li, G. Li, J.-M. Wang, S.-Y. Zhu, and T.-C. Zhang, J. Phys. B 43, 085504 (2010).

[30] A. Miranowicz, K. Bartkiewicz, A. Pathak, J. J. Peřina, Y.-N. Chen, and F. Nori, Phys. Rev. A 91, 042309 (2015).

[31] R. J. Glauber, Phys. Rev. 130, 2529 (1963).

[32] U. M. Titulaer and R. J. Glauber, Phys. Rev. 140, B676 (1965).

[33] L. Mandel, Phys. Scr. 1986, 34 (1986).

[34] W. Vogel and D.-G. Welsch, Quantum Optics, 3rd rev. and extended ed. (Wiley-VCH, Berlin, 2006), p. 520.

[35] L. M. Johansen, Phys. Lett. A 329, 184 (2004).

[36] R. W. Spekkens, Phys. Rev. Lett. 101, 020401 (2008).

[37] T. Kiesel, Phys. Rev. A 87, 062114 (2013).

[38] N. Lütkenhaus and S. M. Barnett, Phys. Rev. A 51, 3340 (1995).

[39] Y. Aharonov, D. Falkoff, E. Lerner, and H. Pendleton, Ann. Phys. (N.Y.) 39, 498 (1966).

[40] M. S. Kim, W. Son, V. Bužek, and P. L. Knight, Phys. Rev. A 65, 032323 (2002).

[41] Wang Xiang-bin, Phys. Rev. A 66, 024303 (2002).

[42] N. Killoran, F. E. S. Steinhoff, and M. B. Plenio, Phys. Rev. Lett. 116, 080402 (2016).

[43] M. M. Wolf, J. Eisert, and M. B. Plenio, Phys. Rev. Lett. 90, 047904 (2003).

[44] S. Olivares and M. G. A. Paris, Phys. Rev. A 80, 032329 (2009).

[45] S. Olivares and M. G. A. Paris, Phys. Rev. Lett. 107, 170505 (2011).

[46] Z. Jiang, M. D. Lang, and C. M. Caves, Phys. Rev. A 88, 044301 (2013).
[47] W. Vogel and J. Sperling, Phys. Rev. A 89, 052302 (2014).

[48] M. Brunelli, C. Benedetti, S. Olivares, A. Ferraro, and M. G. A. Paris, Phys. Rev. A 91, 062315 (2015).

[49] A. Miranowicz, K. Bartkiewicz, N. Lambert, Y.-N. Chen, and F. Nori, Phys. Rev. A 92, 062314 (2015).

[50] A. Mari and J. Eisert, Phys. Rev. Lett. 109, 230503 (2012).

[51] V. Veitch, N. Wiebe, C. Ferrie, and J. Emerson, New J. Phys. 15, 013037 (2013).

[52] R. L. Hudson, Rep. Math. Phys. 6, 249 (1974).

[53] M. G. Genoni, M. G. A. Paris, and K. Banaszek, Phys. Rev. A 78, 060303 (2008).

[54] A. Ferraro, S. Olivares, and M. G. A. Paris, Gaussian States in Continuous Variable Quantum Information (Bibliopolis, Naples, Italy, 2005).

[55] G. W. Bund and M. C. Tijero, Phys. Rev. A 61, 052114 (2000).

[56] L. G. Villanueva, R. B. Karabalin, M. H. Matheny, E. Kenig, M. C. Cross, and M. L. Roukes, Nano Lett. 11, 5054 (2011).

[57] A. N. Cleland, Foundations of Nanomechanics: From SolidState Theory to Device Applications (Springer, Berlin, 2003).

[58] P. M. Morse, Phys. Rev. 34, 57 (1929).

[59] A. Frank, A. L. Rivera, and K. B. Wolf, Phys. Rev. A 61, 054102 (2000).

[60] T. Hahn, Comput. Phys. Commun. 176, 712 (2007).

[61] S. Flügge, Practical Quantum Mechanics (Springer, Berlin, 1971).

[62] B. Y. Tong, Solid State Commun. 104, 679 (1997).

[63] B. Y. Tong and N. Kiriushcheva, Phys. Lett. A 229, 49 (1997).

[64] J. Radovanović, V. Milanović, Z. Ikonić, and D. Indjin, Phys. Lett. A 269, 179 (2000).

[65] H. Yildirim and M. Tomak, Phys. Rev. B 72, 115340 (2005).

[66] H. Yildirim and M. Tomak, J. Appl. Phys. 99, 093103 (2006).

[67] J. J. Sakurai and J. Napolitano, Modern Quantum Mechanics, 2nd ed. (Addison-Wesley, Boston, 2011).

[68] K. E. Cahill and R. J. Glauber, Phys. Rev. 177, 1882 (1969).

[69] S. M. Barnett and P. M. Radmore, Methods in Theoretical Quantum Optics (Oxford University Press, Oxford, 1997). 